\title{
EFFICACY OF TRANSABDOMINAL SONOGRAPHY IN LOCALISATION AND CHARACTERISATION OF GASTROINTESTINAL MALIGNANCIES IN PATIENTS WITH VAGUE ABDOMINAL SYMPTOMS
}

\author{
Rudresh Hiremath', Siddappa Naik2, Harish Reddy T3, Jebin Ibrahim4 ${ }^{4}$ Haritha Ch'5 ${ }^{5}$ Rushit Shah ${ }^{6}$
}

1 Professor, Department of Radiodiagnosis, KVG Medical College, Sullia.

${ }^{2}$ Senior Resident, Department of Radiodiagnosis, KVG Medical College, Sullia.

3 Senior Resident, Department of Radiodiagnosis, KVG Medical College, Sullia.

4 Senior Resident, Department of Radiodiagnosis, KVG Medical College, Sullia.

5 Senior Resident, Department of Radiodiagnosis, KVG Medical College, Sullia.

${ }^{6}$ Senior Resident, Department of Radiodiagnosis, KVG Medical College, Sullia.

\section{ABSTRACT}

Bowel related symptoms often necessitate numerous radiographic investigations, but ultrasonography is not often chosen as initial imaging technique. Recently due to metamorphosis of high resolution ultrasound probes and software, transabdominal sonography is an upcoming screening modality to rule out bowel malignancy.

\section{AIMS AND OBJECTIVES OF THE STUDY}

To evaluate the diagnostic performance and role of trans-abdominal ultrasonography in excluding the malignancy of bowel in the initial workup of patients with abdominal complaints. To correlate the ultrasonography features with histopathological examination.

\section{METHOD OF COLLECTION OF DATA}

Total of 52 patients presenting to the various departments of KVG Medical College, Sullia, DK, with clinically palpable bowel mass, abdominal pain, haematemesis, jaundice, altered bowel habits, are included in the study. All included patients are evaluated by transabdominal ultrasonography and if the characteristic bowel lesions are noted like wall thickening, irregular margins, marked hypoechogenity, degree of preservation of gut signature or any secondary, then the patient is selected for study. The final diagnosis of lesion as malignant or non-malignant is confirmed after the patient has undergone various procedures for tissue diagnosis.

\section{RESULTS}

In current study, $71.2 \%$ of cases were male and $28.8 \%$ female. $94.2 \%$ of cases were malignant and $5.8 \%$ cases were inflammatory/infectious lesions. The sensitivity, positive likelihood ratio, disease prevalence \& positive predictive value for the present study were 100\% (Confidence interval 95\%-92.68 to 100\%), 1 (95\% confidence interval, 1.00 to 1.00 ), $94.23 \%$ (95\% confidence interval, $84.3 \%$ to $98.73 \%$ ) and $94.23 \%$ (Confidence interval $84.3 \%$ to $98.73 \%$ ). With the application of Chi square test, the relationship between the transabdominal ultrasonography and histopathological confirmation of bowel malignancy was highly significant with $\mathrm{P}$ value of 0.002 .

\section{CONCLUSION}

Transabdominal ultrasonography has rapidly become the imaging modality of choice for small and large bowel wall pathologies.

\section{KEYWORDS}

Bowel Malignancy, Transabdominal Sonography, Loss of Gut Signature,

HOW TO CITE THIS ARTICLE: Hiremath R, Naik S, Reddy HT, et al. Efficacy of transabdominal sonography in localisation and characterisation of gastrointestinal malignancies in patients with vague abdominal symptoms. J. Evolution Med. Dent. Sci. 2016;5(73):5404-5409, DOI: 10.14260/jemds/2016/1226

\section{INTRODUCTION}

Two to three decades ago, only few radiologists, such as F. Weill and other pioneers in the field, believed in the diagnostic potential of transabdominal ultrasonography for the study of gastrointestinal tract. Rapid progress in computer technology and transducer design has opened totally new horizons for ultrasonography in the gastrointestinal tract pathology in

Financial or Other, Competing Interest: None.

Submission 25-07-2016, Peer Review 28-08-2016,

Acceptance 02-09-2016, Published 12-09-2016.

Corresponding Author:

Dr. Rudresh Hiremath,

Professor,

Department of Radiodiagnosis,

KVG Medical College, Sullia-574327,

Dakshina Kannada, Karnataka.

E-mail: drrudresh76@yahoo.co.in

DOI: $10.14260 /$ jemds/2016/1226 children and adults.[1] Gastric and colorectal cancers comprise three-fourths of all cancers of the gastrointestinal (GI) tract. On ultrasonography, abnormal bowel lesions may appear as fungating mass with eccentrically located bowel lumen (Pseudokidney sign) or symmetrical or asymmetrical, circumferential thickening of the colonic wall (Target sign). There has recently been a rapid change and advancement in diagnosis of cancers of the GI tract. However, almost all methods require a long period and are invasive and costly. $[2,3]$ Today, ultrasonography is the first preferred imaging method in diagnosis of many diseases due to its advantages of accessibility, cost efficiency and non-invasiveness as compared to the other imaging methods. ${ }^{[4]}$ In the recent years, its reliability and preferability has been remarkably increased with the use of high frequency probes. The aim of our study is to emphasise the role of transabdominal ultrasonography in the diagnosis of gastrointestinal malignancies with substantial sensitivity and to highlight characteristic sonographic 
features. Ultrasound is commonly used before endoscopic interventions as it is non-invasive and easily-accessible.

\section{OBJECTIVES OF THE STUDY}

To evaluate the diagnostic performance and role of transabdominal ultrasonography in excluding the malignancy of bowel in the initial workup of patients with abdominal complaints. To correlate the ultrasonography features of bowel neoplasm with histopathological examination.

\section{MATERIALS AND METHODS}

This is prospective cross sectional study conducted in the Department of Radiodiagnosis, KVG Medical College and Hospital, Kurunjibag, Sullia. Study was carried out for a period of 18 months from November 2012 to May 2014. Total of 52 patients were selected in the study ailing from vague abdominal complaints from various clinical departments. Patients below the age of 12 years are excluded from the study.

\section{Examination of Cases}

All the subjects were informed about the study in their local language and written consent was taken from them. Thorough clinical history and laboratory investigations were elicited from the subjects. Examination technique and harmlessness of sonography was explained to the subjects and were taken into the confidence. Patients were made to lie supine on ultrasound couch with relaxed abdomen.

Transabdominal sonographies were performed by both curvilinear and linear transducers using GE Voluson 730 expert, Siemens Sonoline G 50 and Mindray ultrasonographic machines.

Sonographic examination is commenced with curvilinear transducer for thorough solid visceral and urinary bladder examination. Then, compression technique is performed across the midline to explore the aorta, IVC and retroperitoneum for lymphadenopathy. Examination of the GIT starts with systemic survey using curvilinear probe to get an overview and attempt was made to pick the bowel pathology.

Subsequently, linear high frequency probe is used with graded compression technique to explore the gastrointestinal tract. For the visualisation of the stomach, longitudinal and transverse sections through the upper abdomen was performed. Subxiphoidal view is taken to look for gastrooesophageal junction and other various hiatuses. The small bowel loops were scanned systematically by parallel overlapping lanes- mowing the lawn technique. Finally, the large bowel and proximal part of sigmoid were examined in transverse section along their respective compartments. Initially, caecum and ascending colon were traced up to the hepatic flexure, then transverse colon up to the splenic flexure and eventually descending colon and proximal sigmoid colon. Rectum was assessed by curvilinear probe with full urinary bladder.

Wall thickness of the alimentary tract differs from part to part and depends largely on the state of distension or contraction. Under normal conditions, stomach wall thickness measures from 3-6 mm, small bowel from 1-3 mm, and the colon from $0.5-4 \mathrm{~mm}$. A contracted intestinal segment should not be misinterpreted as a thickened wall. High-resolution transducers usually allow for visualisation of five concentric layers of the normal gastric or bowel wall. Five distinct layers can be depicted on sonography: An inner hyperechoic layer, which is the interface between the mucosa and the bowel contents; a second hypoechoic layer, which is the deep mucosa; a third hyperechoic layer, which is the submucosa; a fourth hypoechoic layer, which is the muscle proper; and a last outer hyperechoic layer, which is the serosa and the serosal fat.[1]

The normal stomach wall measures 3 to $5 \mathrm{~mm}$; however, wall thickness up to $7 \mathrm{~mm}$ is considered within normal limits because adequate distension is difficult to achieve on conventional sonography.[5] Thickening can be due to neoplastic or inflammatory causes, and thickening of greater than $1 \mathrm{~cm}$ is generally considered to be due to malignancy. Visualisation of the stomach can be improved by using a water load.[6]

The appearance of the small bowel on sonography depends not only on the structure of the individual segment, but also more importantly on its contents and degree of distension. The bowel may be collapsed, containing only a small amount of mucus or may contain fluid or gas. The mucus pattern is seen as a target appearance with a highly reflective core of mucus. The fluid pattern gives a tubular appearance on a longitudinal section and a rounded pattern on a cross section. The jejunum has valvulae conniventes, which gives a ladder pattern, and the ileum has smooth, featureless walls. The site of the involved bowel must also be inferred from the location of the bowel loop. The large bowel has a similar appearance; however, it can be distinguished by the location in the paracolic regions and the presence of haustra.[7]

Subjects with sonological features like wall thickening, irregular margins, marked hypoechogenicity of walls, and loss of gut signature and locoregional metastatic lymph nodes were considered as malignant bowel pathology and were included in the study. Positive subjects were also assessed for the presence of mesenteric, hepatic and splenic metastases.

These patients were subjected for the final assessment by endoscopy or colonoscopy and their guided biopsy. Some of the patients underwent CT abdomen and pelvis for confirmation and staging of the disease. Most of the patients who were eligible and fit were operated in our institute and rest were referred to the higher oncology centres. All 52 patients were followed up with their histopathological examination reports.

Sonographic data were analysed and tabulated and compared with colonoscopic/endoscopic/postoperative histopathological examinations. Sensitivity, positive likelihood ratio and positive predictive value of transabdominal ultrasonography examination in gastrointestinal malignancy were analysed.

\section{RESULTS}

Total of fifty two patients suffering from vague abdominal complaints with transabdominal ultrasonographic features of gastrointestinal malignancies were evaluated, in which 14 were female and 38 were male patients. The minimum age of the subjects suffering from bowel cancer was 25 years and maximum age of subject was 83 years. The mean age group in this study was 53.94 years with standard deviation of 13.646 . According to the current study subjects in $7^{\text {th }}$ decade followed by $6^{\text {th }}$ decade are more affected by the gastrointestinal malignancies. On combining the age and sex distribution of bowel malignancies in the current study, maximum number of 
females were affected in the age group of 36-45 years, on the contrary males are affected in the age group of 56 to 65 years. Most common symptoms with which patient presented for ultrasonographic examination was abdominal pain followed by weakness and altered bowel habits. Eight patients suffering from the gastro-oesophageal junction malignancy and gastric malignancy presented with complaints of dysphagia.

Colonic carcinoma topped the table followed by the gastric malignancy in our study accounting for 23 and 13 respectively. Least number of malignancies detected by transabdominal sonography was lower oesophagus and GE junction and small bowel neoplasm accounting for 3 and 6 respectively. Seven cases were rectal malignancies. Colon followed by gastric malignancies were most frequent gastrointestinal malignancies in male subjects whereas gastric malignancy topped the list in females followed by the large bowel.

All 52 patients who underwent preoperative or postoperative histopathological examinations showed 49 malignant lesions and three were benign lesions. Among the malignant bowel lesions, most common histological variety was adenocarcinoma followed by lymphoma.

The sensitivity, positive likelihood ratio, disease prevalence \& positive predictive value for the present study were $100 \%$ (Confidence interval 95\%-92.68 to 100\%), 1 (95\% confidence interval, 1.00 to 1.00 ), $94.23 \%$ (95\% confidence interval, $84.3 \%$ to $98.73 \%$ ) and $94.23 \%$ (Confidence interval $84.3 \%$ to $98.73 \%$ ). With the application of Chi square test, the relationship between the transabdominal ultrasonography and histopathological confirmation of bowel malignancy was highly significant with $\mathrm{P}$ value of 0.002 .
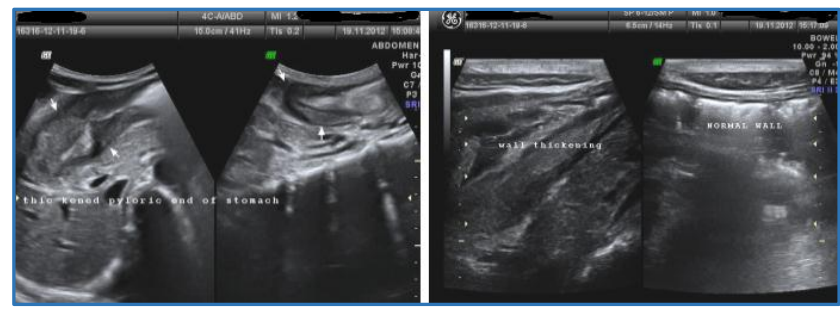

Fig. 1A: Diffuse circumferential wall thickening of pylorus of stomach. High resolution ultrasonography shows normal stomach wall and thickened pyloric antrum with loss of gut signature.
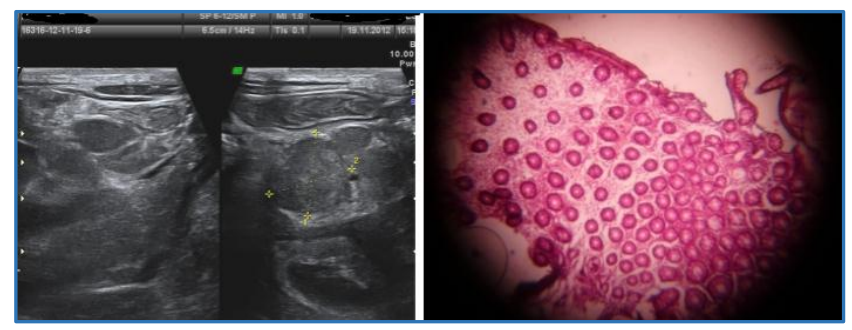

Fig. 1B: Axial high resolution ultrasound depicting locoregional metastatic lymphadenopathy. Histopathological section confirming adenocarcinoma of stomach.
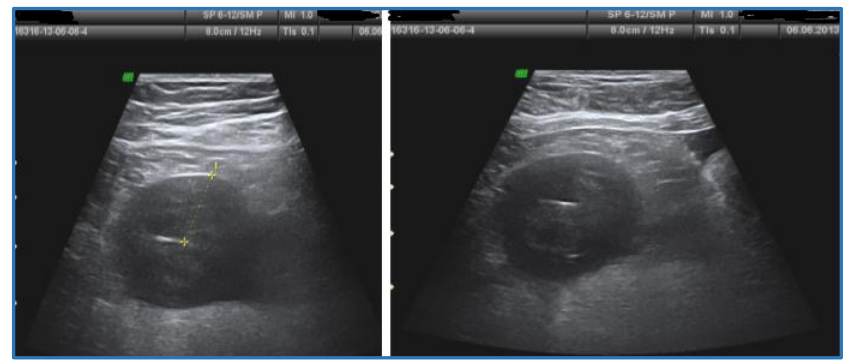

Fig. 2A: Diffuse circumferential wall thickening of jejunal loops with marked hypoechogenicity causing significant luminal narrowing. Minimal ascites present.

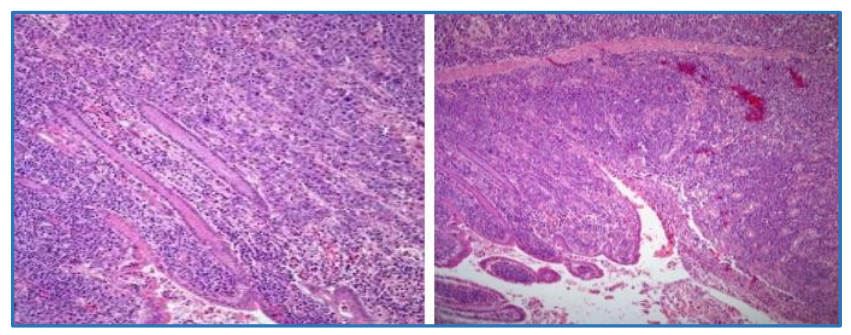

Fig. 2B: Histopathological sections confirming the diagnosis of lymphoma of jejunum.
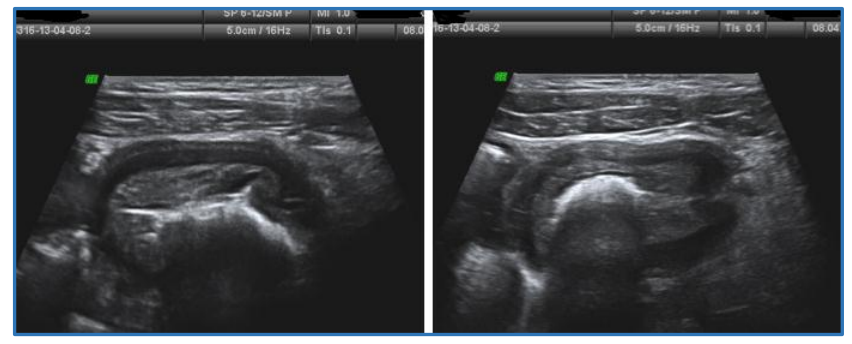

Fig. 3A: Axial section shows diffuse circumferential wall thickening of rectosigmoid junction with loss of GUT signature causing significant luminal narrowing
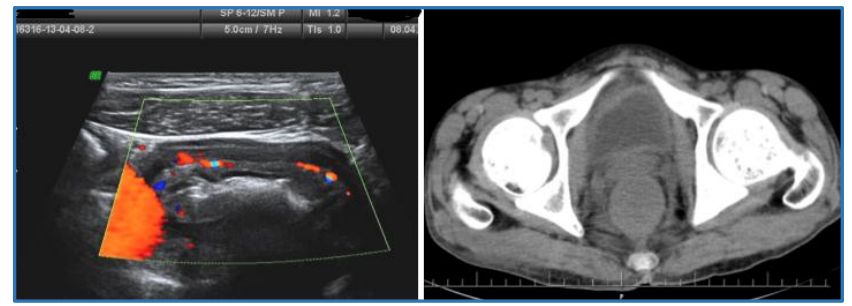

Fig. 3B: On colour Doppler, the mass lesion shows increased vascularity. Non-enhanced axial CT section of pelvis shows diffuse circumferential wall thickening of rectum causing luminal narrowing.
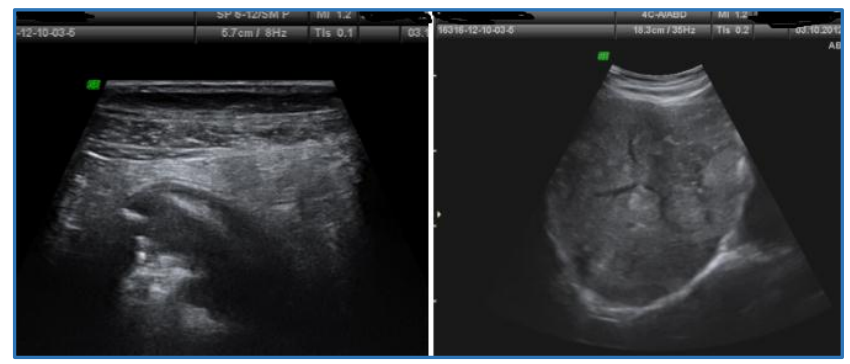

Fig. 4A: Axial section of hepatic flexure of colon shows diffuse circumferential wall thickening with loss of GUT signature. Multiple rounded heteroechoic, predominantly hyperechoic nodules are studded in both lobes of liver suggestive of hepatic metastasis. 


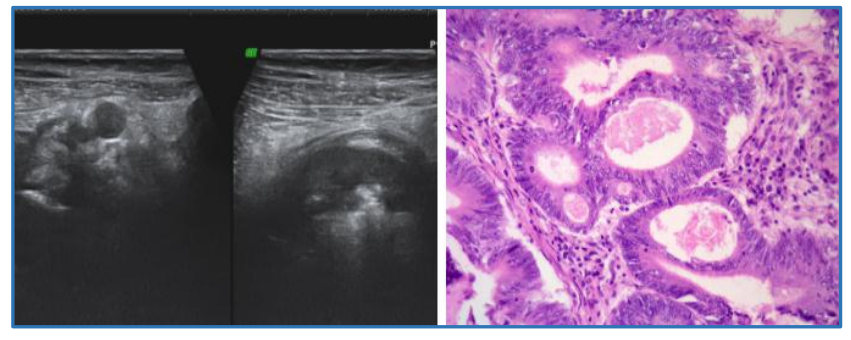

Figure 4B. Axial section showing locoregional lymphadenopathy. Histopathological bloc confirms the diagnosis of adenocarcinoma of colon
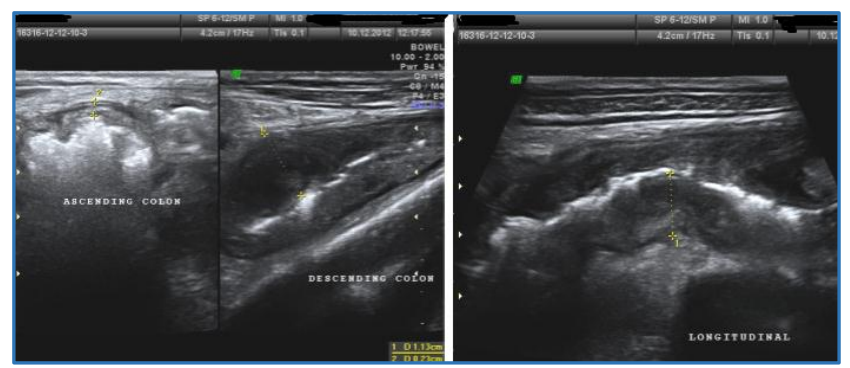

Fig. 5A: Diffuse circumferential wall thickening of descending colon is depicted in transverse section of sonographic images. The first image shows the wall thickening difference between the normal ascending colon and the descending colon.
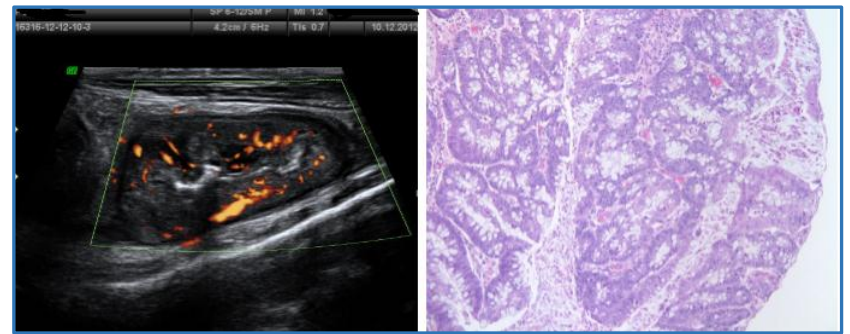

Fig. 5B: On power Doppler, the mass lesion shows hypervascularity. Histopathological section confirming the diagnosis of adenocarcinoma of colon.

\section{DISCUSSION}

There is remarkable imaging development in the diagnosis of gastrointestinal diseases, from the era of diagnosing bowel pathologies on conventional barium studies to the capsule endoscopy where entire small \& large bowel loops are screened through the small capsule shaped endoscope. Technological advancements in the manufacture of newer CT machines and high Tesla magnetic resonance imaging has helped immensely in diagnosing bowel pathologies with techniques like CT enteroclysis, MR enteroclysis, virtual colonoscopy and MR colonography. Isotropic imaging with newer multislice CT \& brilliant software helps in reformations of images in all directions and helps to see the colon in entirety by the techniques like virtual dissection. But most of the patients with typical bowel symptoms or with vague abdominal complaints land in the sonographic world for early screening and diagnosis.

Two to three decades ago only few radiologists, such as F. Weill and other pioneers in the field, believed in the diagnostic potential of transabdominal ultrasonography for the study of gastrointestinal tract. The main applications of ultrasound were confined to the study of solid visceral organs, the female pelvis and obstetrics. Rapid progress in computer technology and in transducer design has opened totally new horizons for USG in the gastrointestinal tract pathology in children and adults.[1]

In the current study, 49 subjects were ailing from gastrointestinal malignancies including $73.4 \%$ (36) of males and $26.6 \%$ (13) of females. Similar study conducted in Turkey showed $62.5 \%$ male patients and $37.5 \%$ of female patients suffering from gastrointestinal cancers. ${ }^{[8]}$

The most common symptom in the study was weakness secondary to anaemia followed by abdominal pain. In colorectal cancer, most common complaint was abdominal pain followed by altered bowel habits and in gastric carcinoma (Figure 1), abdominal pain was common. In the study conducted by Eda Parlak et al, common symptom was abdominal pain. ${ }^{[8]}$

Eda Parlak et al in his study showed that anaemia was the most frequent laboratory result in patients suffering from gastrointestinal malignancies.[8] Similar results were also present in our study, $91.8 \%$ (45) of patients showed haemoglobin below the level of normal limits respective for the both sexes.

The current study showed colorectal carcinoma (Figures 3 , $4 \& 5$ ) topped the list with 21 cases followed by gastric carcinoma. Among the positive cases for colorectal carcinoma, $18(81.8 \%)$ cases were males and $3(13.6 \%)$ were females. Conventional transabdominal ultrasonography showed the sensitivity of $100 \%$ in diagnosing colorectal carcinoma in the study.

Although the studies carried out to date are scarce involving few patients, some data on the high sensitivity of abdominal ultrasound in the diagnosis of the colorectal carcinoma already exists. In their study done in 1991, Rutgeerts et al observed a high sensitivity of $95 \%$ and specificity of $67 \%$. In 95 consecutive patients with proven colonic carcinoma, the tumour was detected by routine sonography in only half of the patients. However, in 22 out of 23 tumours of the right colon, a correct diagnosis was suggested by sonography (sensitivity 95.5\%). Although not suitable as a first choice screening procedure for colorectal cancer, routine abdominal ultrasound can detect even nonsuspected colonic tumours, especially in the ascending colon. [9]

In the study carried out by Richardson et al in 1998, fiftyfour patients with known or suspected colonic carcinoma were referred for abdominal ultrasonography. A single radiologist performed scans on these patients and the site of any colonic mass or wall thickening considered to be consistent with a colonic carcinoma was reported. All carcinomas were confirmed by histology on tissue obtained at colonoscopy or surgery against which the ultrasonographic diagnosis was compared. Colonic masses detected in patients undergoing routine abdominal ultrasonography for abdominal symptoms were also reported. Forty-five of the 54 patients referred had colonic carcinoma and abdominal ultrasonography detected 43 of the tumours and correctly identified the sites of 41 . The sensitivity, specificity and accuracy of abdominal ultrasonography in the detection of colonic tumours considered to be consistent with a colonic carcinoma were 96, 67 and 91 percent respectively. In our study also the sensitivity and positive predictive value is $94.23 \%$ with a sensitivity of $100 \%$. Richardson et al concluded 
that abdominal ultrasonography may detect a colonic mass or wall thickening consistent with a colonic carcinoma with a high degree of accuracy and may be useful when barium enema or colonoscopy is not possible.[10]

Martinez Ares D et al in the year 2005 carried out a study with a statistical analysis to establish factors predictive of colorectal carcinoma and they included following variables like age, sex, presence of anaemia, lower gastrointestinal tract haemorrhage and altered bowel habits. 145 patients were included in the study and cancer was diagnosed in 42 patients. They found that in the diagnosis of colon cancer abdominal ultrasound presents a sensitivity of $79.06 \%$, specificity of $92.15 \%$ and a PPV of $80.9 \%$. By excluding the rectal malignancy which cannot be adequately evaluated by means of ultrasound, the figures increased. They also found that the presence of anaemia is strongly associated with colon cancer.[11] In the current study, we found that out of 22 colorectal malignancy patients, 21 patients presented with significant anaemia.

Hydrocolonic sonography is a novel technique which consists of transabdominal sonography after retrograde instillation of saline solution into the colon. The technique was initially developed by Limberg. The sensitivity of hydrocolonic sonography in the detection of colorectal carcinoma in the various studies oscillates between 83 to $97 \%$ and specificity is in around 98\%. The precision of $\mathrm{T}$ staging of colorectal carcinoma with hydrocolonic sonography is around 70 to 98\%.[12,13,14] On comparison, current study and study conducted by D Martinez A et al (After excluding rectal carcinoma) showed similar or much higher sensitivity on conventional ultrasonography. Moreover conventional ultrasound is less tedious, less time consuming and comfortable to patients than hydrocolonic sonography. Hydrocolonic sonography technique as routine procedure in each and every patient with colonic pathology is debatable; however, its utility in T staging of tumour prior to the surgery is worth mentioning.

There were 14 gastric carcinomas in the present study. Conventional ultrasonography showed $100 \%$ sensitivity in picking up the lesions with confidence interval of 92.68 to $100 \%$. Similar study conducted in Maulana Azad Medical College by S Singh and V Chowdhury showed 100\% sensitivity in diagnosing gastric carcinoma by high resolution transabdominal sonography.[15] In another study by Hamdy A Mokareb et al concluded that gastric wall thickness on transabdominal sonography of more than $10 \mathrm{~mm}$ is the best cut-off point for prediction of gastric malignant tumours with sensitivity of $94.4 \%$, specificity of $100 \%$ and PPV of $100 \%$ and NPV of $80 \% .{ }^{[16]}$

The study done by Martinez Ares D in the year 2008 concludes that ultrasound has high diagnostic accuracy in finding the bowel malignancies. They did the study recruiting 79 patients and found that among the cancer patients 12 were colon cancers and 5 were stomach malignancies. The figures for sensitivity, specificity, PPV, Negative Predictive Value (NPV) and global accuracy of ultrasonography were $80 \%$, $98.6 \%, 80 \%, 98.6 \%$, and $97.4 \%$, respectively, for gastric cancer, while these figures were $100 \%, 94.5 \%, 80 \%, 100 \%$, and $95.5 \%$, respectively, for colon cancer. In the present study with 49 malignant cases, 22 cases were positive for colon carcinoma and one case was diagnosed as infective aetiology by HPR. The figures for colon carcinoma in the current study are sensitivity $100 \%$, positive likelihood ratio of 1 , disease prevalence of $95.65 \%$ and positive predictive value of $95.65 \%$. Totally, 14 cases were diagnosed as gastric carcinoma by transabdominal ultrasonography with sensitivity of $100 \%$ \& positive predictive value of $100 \%$.

Malignant tumours in the small intestine represent only $2 \%$ of gastrointestinal cancers. The most frequent small bowel cancers are adenocarcinoma (30-50\%), followed by carcinoids (25-30\%) and lymphoma (15-20\%). Mesenchymal tumours predominate in jejunum, adenocarcinoma in duodenum and carcinoid \& lymphoma in ileum.[17] As result of low number of patients, there have been few studies confirming the transabdominal characters of small bowel tumours. In the current study, there were total six cases diagnosed as small bowel tumours, four in males and two in females. Out of six cases, four were turned malignant on histopathological examination, three lymphomas (Figure 2) and one adenocarcinoma of duodenum.

Seven patients out of 49 cases showed multiple hepatic metastases with target appearance, three from gastric carcinoma and four from colonic carcinoma. Prospective study conducted by Eda Parlak showed $26 \%$ of patients in his study showed metastatic hepatic nodules.[8]

Out of 52 subjects, 49 were confirmed as malignant on histopathological examination. The sensitivity of the study was $100 \%$ (With confidence interval of $92.6 \%$ to $100 \%$ ), positive predictive value of $94.23 \%$ and positive likelihood ratio of 1 . Similar study conducted by Eda Parlak et al in 40 patients, the sensitivity of the study was $71.4 \%$, specificity of $75.7 \%$, positive predictive value of $65.2 \%$ and negative predictive value of $19.3 \%{ }^{[8]}$ Even though the number of patients in both studies was near, the sensitivity \& PPV of our study were much higher than study conducted by Eda Parlak et al. We could not calculate specificity and negative predictive value of our study as we have excluded the bowel pathologies with inflammatory changes. Most of the excluded cases were followed up and were turned out to be benign.

After the extensive elaboration of results of current study and comparison with previous studies, it can be said that transabdominal ultrasonography is readily available and easily performed imaging modality for the diagnosis and screening of gastrointestinal malignancies. It has simple technique, is less time consuming and compliable for the patients, moreover no worry of radiation hazards as with CT or barium studies. Transabdominal sonography with proper technique and experienced hands not only provides high sensitivity and specificity in diagnosing the gastrointestinal tumours but also aids in the $\mathrm{T}$ staging in colonic tumours prior to the surgery.

\section{CONCLUSION}

Proper technique with good skills aids in the diagnosis of gastrointestinal pathologies including bowel malignancies by transabdominal sonography. Transabdominal sonography not only diagnoses bowel malignancies but also detects locoregional lymphadenopathy and metastatic lesions in the liver and spleen. Transabdominal ultrasonography should not be restricted to the solid organs, pelvis and pelvic organs but must include thorough screening of small and large bowel loops by mowing the abdomen in each and every patient with vague abdominal complaints. 


\section{REFERENCES}

1. Maconi G, Porro GB. Ultrasound of the gastrointestinal tract diagnostic imaging. Germany, Springer 2007: 127-51.

2. Martínez-Ares D, Alonso-Aguirre PA, Souto-Ruzo J, et al. Ultrasonography is an accurate technique for the diagnosis of gastrointestinal tumors in patients without localizing symptoms. Rev Esp Enferm Dig 2009;101(11):773-86.

3. Loftus WK, Metreweli C, Sung JJ, et al. Ultrasound, CT and colonoscopy of colonic cancer. $\mathrm{Br} J$ Radiol 1999;72(854):144-8.

4. Bierig SM, Jones A. Accuracy and cost comparison of ultrasound versus alternative imaging modalities, including CT, MR, PET, and angiography. Journal of diagnostic medical sonography 2009;25(3):138-44.

5. Rapaccini GL, Aliotta A, Pompili M, et al. Gastric wall thickness in normal and neoplastic subjects: a prospective study performed by abdominal ultrasound. Gastrointest Radiol 1988;13(3):197-9.

6. Derchi LE, Biggi E, Neumaier CE, et al. Ultrasonographic appearances of gastric cancer. $\mathrm{Br} \mathrm{J}$ Radiol 1983;56(666):365-70.

7. Peck R. The small bowel. In: Meire HB, Cosgrove DO, Dentoury $\mathrm{KC}$, et al (eds). Abdominal and general ultrasound. $2^{\text {nd }}$ ed. Philadelphia, PA: Churchill Livingstone 2002:823-64.

8. Parlak E, Mustafa Y, Çekin AH, et al. Role of ultrasonography in the diagnosis of cancers of gastrointestinal tract. Journal of clinical and analytical medicine 2013;4(5):377-80.
9. Rutgeerts LJ, Verbanck JJ, Crape AW, et al. Detection of colorectal cancer by routine ultrasound. J Belge Radiol 1991;74(1):11-3.

10. Richardson NG, Heriot AG, Kumar D, et al. Abdominal ultrasonography in the diagnosis of colonic cancer. $\mathrm{Br} \mathrm{J}$ Surg 1998;85(4):530-3.

11. Martínez AD, Alonso-Aguirre PA, Martín-Granizo BI, et al. Usefulness of ultrasonography in diagnosing patients suspect for digestive tract neoplasms. Rev Esp Enferm Dig 2008;100(9):545-51.

12. Limberg B. Diagnosis of colonic tumors and chronic inflammatory colonic diseases by hydrocolonic sonography. Radiologe 1993;33(7):407-11.

13. Hernández-Socorro CR, Guerra C, Hernández-Romero J, et al. Colorectal carcinomas: diagnosis and preoperative staging by hydrocolonic sonography. Surgery 1995;117(6):609-15.

14. Limberg B. Diagnosis and staging of colonic tumors by conventional abdominal sonography as compared with hydrocolonic sonography. N Engl J Med 1992;327(2): 65-9.

15. Singh S, Chowdary V. Efficacy of high resolution transabdominal sonography of fluid filled stomach in the evaluation of gastric carcinoma. IJRI 2005;15(4):421-6.

16. Mokareb HA, El-Hini SH, Abd El Monem HM, et al. The value of gastric wall thickening and loss of gastric wall layering on transabdominal ultrasonography (TAS) in prediction of gastric cancer. El-Minia Med Bull $2009 ; 20(1)$.

17. Nylund K, Odegaard S, Hausken T, et al. Sonography of small intestine. World J Gastroenterol 2009; 15(11): 1319-30. 